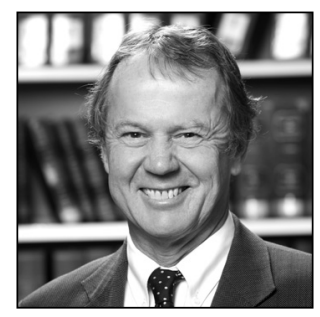

Michael Sachs

Prof. Dr.

Universität zu Köln

\title{
Verfassungsrechtliche Determinanten der öffentlichen Verwaltung in Deutschland'
}

\section{Einführung}

Zum fünften Thema dieser Tagung, ,Über die Determinanten der modernen Staatsverwaltung', könnte man die unterschiedlichsten Dinge sagen - geschichtliche Traditionen, soziologische Gegebenheiten, politische und ökonomische Rahmenbedingungen, die zu erledigenden Aufgaben: Sie alle und weitere Faktoren bestimmen mit darüber, was moderne Staatsverwaltung tut und wie sie beschaffen ist. Für mich als Juristen liegt es nahe, den Blick auf rechtliche Determinanten zu werfen; von diesen haben unbeschadet unionsrechtlicher Ingerenzen die Vorgaben der Verfassung die größte Bedeutung. Über diese will ich Ihnen mit Blick auf das mir vertraute Deutschland berichten und hoffe, dass dies für Sie aus rechtsvergleichender Perspektive von Interesse ist.

Estland ist als Einheitsstaat mit gesetzlich festgelegter territorialer Verwaltungseinteilung verfasst (§ 2 Abs. 2 der Verfassung von $1992^{*}{ }^{2}$ ); Deutschland ist demgegenüber ein Bundesstaat, der Bund mit seinem ,Grundgesetz' und die ihm als seine Gliedstaaten angehörenden Länder haben ihre je eigenen Verfassungen, die als ,Determinanten' zu berücksichtigen sind. Die öffentliche Verwaltung in Deutschland findet grundsätzlich auf der Ebene der Länder statt; die Landesverwaltungen führen die jeweiligen Landesgesetze, aber prinzipiell auch die Bundesgesetze aus. Dabei hat der Bund nach Art. 84, 85 Grundgesetz (GG) unterschiedlich weit gehende Möglichkeiten der Aufsicht über die Länder. Eigene Verwaltung des Bundes gibt es nur in einzeln im Grundgesetz aufgeführten Fällen. Sie ist teils nur nach Maßgabe von Bundesgesetzen zugelassen - wie etwa bei Aufgaben der Schifffahrt in Art. 89 Abs. 2 Satz 2 GG -, teils verfassungsunmittelbar vorgeschrieben - wie etwa bei der Bundeswehrverwaltung in Art. 87b Abs. 1 Satz 1 GG. Für die Bundesverwaltung kommt als Determinante nur die Bundesverfassung, das Grundgesetz, in Betracht; dieses ist in vielem aber auch für Fragen der Landesverwaltung maßgeblich, die daneben zudem von der jeweiligen Landesverfassung determiniert wird. Insoweit besteht eine doppelte verfassungsrechtliche Determinierung; im allerdings seltenen Konfliktfall setzt sich die Bundesverfassung durch, denn ganz allgemein gilt nach Art. 31 GG: Bundesrecht bricht Landesrecht. Daher - und weil Fragen der Bundesstaatlichkeit für Estland wohl von geringerem Interesse sind - werde ich mich im Weiteren vor allem auf die Determinanten aus dem Grundgesetz beziehen.

Für Unterstützung bei der Gestaltung der Fußnoten danke ich meinem Wissenschaftlichen Mitarbeiter Dr. Thomas Blome.

2 Eesti Vabariigi põhiseadus (The Constitution of the Republic of Estonia). - RT 1992, 26, 349 (auf Estnisch). Auf Englisch verfügbar unter: http://www.legaltext.ee/ (30.3.2014). 
Mein Vortragsgegenstand weicht von der Formulierung des Oberthemas, das von moderner Staatsverwaltung spricht, in zwei Punkten ab. Weggelassen habe ich das ,moderne', weil ich mich nur mit der heutigen Lage befassen will, und nicht mit der geschichtlichen Entwicklung in der sog. Moderne. Mit dem Begriff der ,öffentlichen Verwaltung' (statt ,Staatsverwaltung') soll auch die Verwaltung erfasst werden, die durch - gegenüber der Rechtsperson Staat selbständige - Rechtsträger erfolgt, insbesondere solche mit Selbstverwaltungsrechten. Wie in Estland gibt es solche Selbstverwaltungskörperschaften auch in Deutschland zumal auf der örtlichen Ebene, als Gemeinden, auch als Gemeindeverbände (,Gebietskörperschaften'); obwohl diese Kommunen territorial und rechtlich Untergliederungen des jeweiligen Landes sind, garantiert Art. 28 Abs. 2 GG ihnen schon von Bundesverfassungs wegen das Recht der Selbstverwaltung. Dies ist damit den Ländern zwingend vorgegeben, ihre Verfassungen können es nur im Detail näher ausformen. Daneben gibt es auf gesetzlicher Grundlage auf Bundes- und Landesebene sog. ,funktionale Selbstverwaltung' (,Personalkörperschaften') für Rechtsträger, die zur Erfüllung bestimmter Aufgaben (Funktionen) geschaffen sind - wie etwa für die Hochschulen; die Existenz solcher Rechtsträger legt das Grundgesetz nicht fest, ${ }^{*}$ sondern lässt sie allenfalls zu - darauf komme ich mit Blick auf das Demokratieprinzip später zurück. Einstweilen ging es mir nur darum, die Reichweite der hier zu behandelnden öffentlichen Verwaltung aufzuzeigen, die über eine eng verstandene Staatsverwaltung hinausgeht; sie unterliegt insgesamt weitgehend denselben bundesverfassungsrechtlichen Determinanten.

Ich habe das Grundgesetz und die Landesverfassungen für diesen Vortrag einmal nach verwaltungsbezogenen Begriffen wie Verwaltung, Behörden, Beamten, Aufsicht und Ähnlichem durchgesehen und dabei festgestellt, in welchem Ausmaß schon der Verfassungswortlaut sich mit Fragen der Verwaltung befasst. Die Überfülle einschlägiger Bestimmungen schließt es aus, sie auch nur annähernd alle wenigstens anzusprechen. Ich werde mich darum im Weiteren vor allem auf zentrale Elemente des deutschen Verfassungsrechts von übergreifender Bedeutung beziehen. Im Einzelnen möchte ich folgende großen Komplexe jeweils in einigen Facetten ansprechen, nämlich:

- vor allem das Rechtsstaatsprinzip (zu II.),

- damit eng zusammen hängend die Grundrechtsbindung aller Staatsgewalt (zu III.),

- schließlich noch kurz das Demokratieprinzip (zu IV).

$\mathrm{Zu}$ den zentralen, selbst der Verfassungsänderung entzogenen Verfassungsgehalten der Art. 1 und 20 GG gehören daneben noch drei Prinzipien:

- das schon erwähnte Bundesstaatsprinzip soll trotz erheblicher Bedeutung für die Verwaltung im föderalen Deutschland aus den erwähnten Gründen hier nicht näher behandelt werden,

- das republikanische Prinzip wird wohl noch überwiegend auf den Ausschluss eines erblichen, monarchischen Staatsoberhaupts beschränkt verstanden ${ }^{*}{ }^{4}$ und erlangt so für die Verwaltung keine Bedeutung. ${ }^{*}$ Soweit ihm weitergehende Gehalte zugeschrieben werden, sind sie durchweg auch über Rechtsstaat und Demokratie zu erfassen,

- das Sozialstaatsprinzip hat trotz größter praktischer Relevanz sozialer Gesetzgebung und Verwaltung doch nur recht begrenzt juristisch greifbare unmittelbare Rechtswirkungen für die Verwaltung, die keine selbständige Behandlung erfordern.

3 Auch für die in Art. 87 Abs. 2 GG angesprochenen Träger der Sozialversicherung wird die ihnen traditionell zukommende Selbstverwaltung ganz überwiegend nicht als grundgesetzlich vorgeschrieben verstanden, s. nur M. Sachs. - M. Sachs (Hrsg.). Grundgesetz. 6. Aufl. München: Beck 2011, Art. 87 Rdn. 55 mit weiteren Nachweisen (m.w.N.).

4 M. Sachs (Fn. 2), Art. 20 Rdn. 9 f.; H. Dreier. - H. Dreier (Hrsg.). Grundgesetz. Bd. II. 2. Aufl. Tübingen: Mohr Siebeck 2006, Art. 20 (Republik) Rdn. $17 f f$.

5 Bei einem erweiterten Republikverständnis mag zumal das damit verbundene Verständnis des Amtes Bedeutung (auch) für die Verwaltung erlangen können, vgl. etwa J. Isensee. § 15 Staat und Verfassung. - J. Isensee, P. Kirchhof (Hrsg.). Handbuch des Staatsrechts der Bundesrepublik Deutschland. Bd. II. 3. Aufl. Heidelberg: Müller 2004, Rdn. 128ff., 131; R. Gröschner. $\S 23$ Die Republik. - Ibid., Rdn. 62 ff. Jedenfalls die Kernpunkte sind aber in den besonderen Regelungen des Art. 33 Abs. 2, 4 und 5 GG erfasst und können auch anderen Staatsstrukturprinzipien zugeordnet werden. 


\section{Rechtsstaatliche Determinanten öffentlicher Verwaltung}

Das Rechtsstaatsprinzip ${ }^{* 6}$ umfasst eine Fülle von im Grundgesetz ausdrücklich angesprochenen oder von der Verfassungsrechtsprechung entwickelten, nicht immer klar voneinander abzugrenzenden Elementen. Manche betreffen speziell die Gesetzgebung oder die Rechtsprechung, die Gewaltenteilung in Art. 20 Abs. 2 Satz 2 GG spezifisch das Gesamtgefüge der Staatsfunktionen. Viele Elemente des Rechtsstaatsprinzips sind aber auch, manche speziell gerade für die Verwaltung von Bedeutung. Das wichtigste von letzteren ist die in Art. 20 Abs. 3 GG verankerte Gesetzmäßigkeit der Verwaltung, auf die ich zuerst eingehen werde (zu 1.). Sodann ist ein Blick zu werfen auf Kontrollen dieser Gesetzmäßigkeit (zu 2.). Daneben sind allgemeine inhaltliche Anforderungen an das Staatshandeln anzusprechen (zu 3.) und schließlich Vorgaben für Organisation und Verfahren der Verwaltung (zu 4.).

\section{Die Gesetzmäßigkeit der Verwaltung}

Nach Art. 20 Abs. 3 GG wird neben der Rechtsprechung auch die Verwaltung ,an Gesetz und Recht gebunden'. Damit wird an den überkommenen Grundsatz der Gesetzmäßigkeit der Verwaltung angeknüpft, der drei Elemente enthält, nämlich die Bindung an das Gesetz (zu a), den Vorrang des Gesetzes (zu b) und den Vorbehalt des Gesetzes (zu c). ${ }^{*} 7$

Für die Gesetzmäßigkeit der Verwaltung ist das Gesetz dabei einheitlich zu verstehen als materielles Gesetz, meint jede (abstrakt-generelle) Rechtsnorm, neben den durchweg vom Parlament ${ }^{* 8}$ oder Volk $^{*} 9$ erlassenen formellen Gesetzen also auch von Stellen der Exekutive erlassene Rechtsverordnungen und Satzungen. ${ }^{* 10}$ Die Verwaltung bedeutet hier die von Gesetzgebung und Rechtsprechung unterschiedene Staatsfunktion, schließt also die (materielle) Gesetzgebung durch die Verwaltung im organisatorischen Sinne nicht ein. ${ }^{* 11}$ So und nur so ist eine konsistente Begrifflichkeit im Rahmen des Grundsatzes gewahrt, ohne dass die besondere Bedeutung des formellen Gesetzes dadurch in Frage gestellt würde.

\section{a) Zur Bindung an das Gesetz}

Die Bindung der Verwaltung an das Gesetz meint deren Verpflichtung, geltende Normen zu beachten, so zu entscheiden oder sonst tätig zu werden, wie es die einschlägigen Normen gebieten, und nichts zu tun, was sie verbieten. In der Pflicht der Normadressaten, die Norm zu befolgen, liegt deren wesensmäßige Bedeutung; die Bindung der Verwaltung an jede für ihr Verhalten geltende Rechtsnorm ist daher unabweisbar. Dies gilt auch für Rechtsverordnungen und Satzungen; selbst wenn eine Stelle der Verwaltung eine solche Rechtsnorm aufheben kann, bleibt ihre Bindung unberührt, bis dies geschehen ist.

Nicht abschließend gelöst ist bis heute die Frage, ob Verwaltungsbehörden auch dann an Rechtsnormen gebunden sind, wenn diese höherrangigen Rechtsnormen widersprechen. ${ }^{*}{ }^{12}$ Nach meiner Meinung kann es

6 Zur unbefriedigenden Verankerung in Art. 20 GG M. Sachs (Fn. 2), Art. 20 Rdn. 75f.; H. Schulze-Fielitz. - H. Dreier (Hrsg.) (Fn. 3), Art. 20 (Rechtsstaat) Rdn. 40; E. Schmidt-Aßmann. § 26 Der Rechtsstaat. - J. Isensee, P. Kirchhof (Hrsg.) (Fn. 4), Rdn. 3.

7 So nach der noch aus dem 19. Jahrhundert stammenden maßgeblichen Formulierung O. Mayer. Deutsches Verwaltungsrecht. Bd. I. Leipzig: Duncker \& Humblot 1895, S. 76. Heute werden vielfach nur Vorrang und Vorbehalt des Gesetzes angesprochen, vgl. in diesem Sinne etwa F. Reimer. § 9: Das Parlamentsgesetz als Steuerungsmittel und Kontrollmaßstab. - W. HoffmannRiem, E. Schmidt-Aßmann, A. Voßkuhle (Hrsg.). Grundlagen des Verwaltungsrechts. Bd. I: Methoden, Maßstäbe, Aufgaben, Organisation. 2. Aufl. München: Beck 2012, Rdn. 73ff.; H. Maurer. Allgemeines Verwaltungsrecht. 18. Aufl. München: Beck 2011, § 6 Rdn. 2, wobei ersterer dann zugleich auf die Bindung an das Gesetz bezogen wird, was der Klarheit nicht dienlich ist.

8 Auf Bundesebene grundsätzlich durch den Bundestag, Art. 77 Abs. 1 Satz 1 GG, aber stets unter Mitwirkung des Bundesrates, Art. 77, 78 GG; formelle Gesetze können ferner gegen den Willen des Bundestages zustande kommen nach Art. 81 GG im sog. Gesetzgebungsnotstand und im Verteidigungsfall durch Beschluss des Gemeinsamen Ausschusses nach Art. 115e GG.

$9 \quad$ Nur auf der Landesebene aufgrund einschlägiger Regelungen zur Volksgesetzgebung.

10 Strittig; s. dazu nur M. Sachs (Fn. 2), Art. 20 Rdn. 107, 118.

11 Auch dies wird vielfach anders gesehen, vgl. H. Schulze-Fielitz. - H. Dreier (Hrsg.) (Fn. 3), Art. 20 (Rechtsstaat) Rdn. 92.

12 Offen lassend Bundesverfassungsgericht (BVerfG), Beschluss v. 10.12.2009, 1 BvR 3151/o7. - Neue Zeitschrift für Verwaltungsrecht (NVwZ) 2010, 435 (441); Bundesverwaltungsgericht (BVerwG), Urteil v. 31.1.2001, 6 CN 2/oo. - Entschei- 
eine Bindung an solche, wegen des Widerspruchs ja nichtige Normen nicht geben; auch $§ 76$ Abs. 1 Nr. 2 Bundesverfassungsgerichtsgesetz (BVerfGG) ${ }^{*} 13$ geht ohne ersichtliche Bedenken davon aus, dass Verwaltungsbehörden in solchen Fällen Rechtsnormen nicht anwenden.

Trotz umfänglichster Normsetzung bleiben allerdings ungeregelte Bereiche; insoweit kann es eine Bindung der Verwaltung an das Gesetz nicht geben. Neben solchen Bereichen gesetzesfreier Verwaltung gibt es Fälle normativer Regelungen, die nicht abschließend sind, in denen das Gesetz der Verwaltung hinsichtlich der anzuordnenden Rechtsfolgen Ermessen einräumt oder ihr für die Beurteilung tatbestandlicher Voraussetzungen einen Beurteilungsspielraum überlässt. Grenzen ergeben sich einerseits aus den Grundrechten, die nicht pauschal dem Ermessen von Verwaltungsstellen überantwortet werden dürfen, ${ }^{* 14}$ andererseits aus der Rechtsweggarantie, die nicht beliebig überspielt werden darf. ${ }^{*} 15$

\section{b) Zum Vorrang des Gesetzes}

Der Vorrang des Gesetzes ist, wenn man ihn nicht ganz oder teilweise mit der Bindung an das Gesetz gleichsetzt, ${ }^{*} 16$ eine Kollisionsnorm. Diese verbietet, dass ein auf die Begründung von Rechtsfolgen ausgerichteter Rechtsakt der Verwaltung, dessen Inhalt einem Gesetz widerspricht, volle Gültigkeit erlangt. Dies bedeutet allerdings nicht, dass rechtswidrige Rechtsakte der Verwaltung zwangsläufig ungültig sein müssen. Die Rücksicht auf konkurrierende Verfassungsanliegen einer effektiven Verwaltung einerseits, der auch zum Rechtsstaatsprinzip gezählten Rechtssicherheit andererseits lassen vielmehr Raum für gesetzliche Regelungen, die die ideale Fehlerfolge der Nichtigkeit rechtswidriger Verwaltungsrechtsakte ${ }^{*} 17$ relativieren.

So sind nach dem überkommenen, heute in den Verwaltungsverfahrensgesetzen normierten allgemeinen Verwaltungsrecht in Deutschland die weiterhin als Handlungsform der Verwaltung zentral bedeutsamen Verwaltungsakte, wenn sie materiell rechtswidrig sind, trotzdem nur ausnahmsweise nichtig. Sie

dungen des Bundesverwaltungsgerichts (BVerwGE) 112, 373 (380 ff.); Bundesgerichtshof (BGH), Urteil v. 25.3.2004, III ZR 227/O2. - NVwZ 2004, 1143 (1144) m.w.N.; wie hier etwa Oberverwaltungsgericht (OVG) Niedersachsen, Beschluss v. 15.10.1999, 1 M 3614/99. - NVwZ 2000, 1061 ff.; auch H.-D. Horn. - M. Sachs, H. Siekmann (Hrsg.). Der grundrechtsgeprägte Verfassungsstaat - Festschrift für Klaus Stern zum 80. Geburtstag. Leipzig: Duncker \& Humblot 2012, S. 353 ff.; OVG Nordrhein-Westfalen, Urteil v. 30.6.2005, 20 A 3988/03. - Entscheidungen der Oberverwaltungsgerichte für das Land Nordrhein-Westfalen und für das Land Niedersachsen (OVGE) 50, 139 (146), sieht immerhin ,in engen Grenzen' Raum für eine behördliche Normverwerfungskompetenz; anders Bundesfinanzhof (BFH), Urteil v. 12.05.2009, IX R 45/o8. Sammlung der Entscheidungen des BFH (BFHE) 225, 299 (301); K.-F. Gärditz. - K. H. Friauf, W. Höfling (Hrsg.). Berliner Kommentar zum Grundgesetz. Köln: Erich Schmidt Verlag (Losebl.-Ausg.), Art. 20 (6. Teil) (2011) Rdn. 108 ff.; BVerwG, Beschluss v. 22.08.2005, 6 BN 1/05. - Neue Zeitschrift für Verwaltungsrecht Rechtsprechungs-Report (NVwZ-RR) 2006, 36 (37), sieht Beamte ,regelmäßig' auf die Möglichkeit beschränkt, Bedenken gegen die Rechtmäßigkeit einer Norm intern oder in Form der sog. Remonstration anzubringen; BVerwG, Urteil v. 16.10.2007, 7 C 6/o7. - BVerwGE 129, 346 Rdn. 28, hält die behördliche Anwendung einer nichtigen Verordnung für unbeachtlich, weil die Nichtigkeit, nicht offensichtlich' gewesen sei; dagegen aber BVerfG, Beschluss v. 10.12.2009, 1 BvR 3151/07. - NVwZ 2010, 435 Rdn. 80 $f$.

13 Die Bestimmung lautet: „Der Antrag der Bundesregierung, einer Landesregierung oder eines Viertels der Mitglieder des Bundestages gemäß Artikel 93 Abs. 1 Nr. 2 des Grundgesetzes ist nur zulässig, wenn der Antragsteller Bundes- oder Landesrecht für gültig hält, nachdem ein Gericht, eine Verwaltungsbehörde oder ein Organ des Bundes oder eines Landes das Recht als unvereinbar mit dem Grundgesetz oder sonstigem Bundesrecht nicht angewendet hat." Zur Argumentation mit der Vorschrift s. F. Kopp. Das Gesetzes- und Verordnungsprüfungsrecht der Behörden. - Deutsches Verwaltungsblatt (DVBl.) 1983 , S. $825 f$.

14 M. Sachs. - P. Stelkens, H. J. Bonk, M. Sachs (Hrsg.). Verwaltungsverfahrensgesetz. 8. Aufl. München: Beck 2014, § 40 Rdn. 85; K. Stern. Das Staatsrecht der Bundesrepublik Deutschland. Bd. III/1: Allgemeine Lehren der Grundrechte. München: Beck 1988, S. 1353 ff.; H. Maurer (Fn. 6), § 7 Rdn. 23.

15 BVerfG, Beschluss v. 18.06.1997, 2 BvR 483/95 u.a., Strafvollstreckungsüberstellung auf eigenen Wunsch. - BVerfGE 96, 100 (114 ff.); BVerwG, Urteil v. 20.02.1990, 1 C 42/83. - BVerwGE 84, 375 (377 f.); M. Sachs. - P. Stelkens, H. J. Bonk, M. Sachs (Hrsg.) (Fn. 13), § 40 Rdn. 90.

16 Vgl. für dieses verbreitete, von der von Otto Mayers Urversion des Grundsatzes der Gesetzmäßigkeit der Verwaltung abweichende Vorgehen etwa BVerfG, Beschluss v. 28.10.1975, 2 BvR 883/73 u.a., Rechtsschutzverfahren im Strafvollzug. BVerfGE 40, 237 (248f.); BVerfG, Beschluss v. 14.10.2004, 2 BvR 1481/04, Görgülü. - BVerfGE 111, 307 (330); K.-P. Sommermann. - H. v. Mangoldt, F. Klein, C. Starck (Hrsg.). Kommentar zum Grundgesetz. Bd. 2. 6. Aufl. München: Vahlen 2010, Art. 20 Rdn. 270 ff.; G. Robbers. - W. Kahl, C. Waldhoff, C. Walter (Hrsg.). Bonner Kommentar zum Grundgesetz. Heidelberg: Müller (Losebl.-Ausg.), Art. 20 I (2009) Rdn. 2105f.; K.-F. Gärditz. - K. H. Friauf, W. Höfling (Hrsg.) (Fn. 11), Art. 20 (6. Teil) (2011) Rdn. 43; H. Maurer (Fn. 6), § 6 Rdn. 2.

17 Für Gerichtsentscheidungen gilt im Hinblick auf die Sicherung von Rechtsfrieden und Rechtssicherheit Ähnliches, vgl. nur BVerfG, Beschluss v. 7.5.1953, 1 BvL 104/52, Notaufnahmegesetz. - BVerfGE 2, 280 (403f.); BVerfG, Beschluss v. 2.5.2012, 2 BvL 5/10, Ruhegehaltssatz. - BVerfGE 131, 20 (46f.); BGH, Beschluss v. 15.4.2008, 5 StR 635/07. - Entscheidungen des Bundesgerichtshofs in Strafsachen (BGHSt) 52, 213 (216f.); BVerwG, Urteil v. 22.10.2009, 1 C 26.08. - BVerwGE 135, 137 Rdn. 14; M. Sachs (Fn. 2), Art. 20 Rdn. 142. 
sind vielmehr trotz Verstoßes ihres Inhalts gegen das Gesetz regelmäßig zunächst wirksam, unterliegen allerdings der Anfechtung durch die in ihren subjektiven Rechten Verletzten, die ggf. zur behördlichen oder gerichtlichen Aufhebung führt. Unterbleibt die nur befristet mögliche Anfechtung, werden rechtswidrige Verwaltungsakte sogar bestandskräftig, sind ggf. mit ihrem gesetzwidrigen Inhalt endgültig für die Betroffenen verbindlich. Die Verwaltung behält zwar die Möglichkeit, ihren Gesetzesverstoß zu korrigieren, kann aber nur in Ausnahmefällen dazu gezwungen werden.

Sind bei Erlass eines Verwaltungsaktes Vorschriften über die Zuständigkeit, das Verfahren oder die Form verletzt worden, hat ein solcher Verstoß gegen Verhaltensanforderungen der Verwaltung nicht begriffsnotwendig zur Folge, dass neben dem Verwaltungshandeln als solchem auch der resultierende Verwaltungsakt selbst, also das Produkt des Verwaltungshandelns, deswegen als rechtswidrig qualifiziert wird. ${ }^{* 18}$ Diese sog. formelle Rechtswidrigkeit tritt vielmehr nur ein, soweit ein Gesetz dies wegen des Gesetzesverstoßes bei Erlass des Verwaltungsaktes vorsieht. Der Vorrang des Gesetzes selbst gibt weder dies noch die weiteren Konsequenzen solcher Rechtswidrigkeit im Hinblick auf Heilungs- bzw. ausgeschlossene Anfechtungsmöglichkeiten ${ }^{*} 19$ von Verfassungs wegen vor.

Öffentlich-rechtliche Verträge sind bei inhaltlicher Rechtswidrigkeit nicht stets, sondern nur im Rahmen des § 59 Verwaltungsverfahrensgesetz (VwVfG) nichtig; dieser geht zwar deutlich weiter als der enge $\S 44$ VwVfG beim Verwaltungsakt, doch fehlt es für die nicht erfassten Fälle an der flankierenden Anfechtungsmöglichkeit des Vertragspartners. Dieses Defizit wird freilich durch dessen Mitwirkung am Vertragsschluss ${ }^{*}{ }^{20}$ wohl hinreichend kompensiert.

Der Vorrang des formellen Gesetzes vor den untergesetzlichen, von der Verwaltung erlassenen materiellen Gesetzen ist unabhängig von Art. 20 Abs. 3 GG durch die im Grundgesetz angelegte Normenhierarchie sichergestellt.

Über den Vorrang des Gesetzes hinaus führt die rechtsstaatliche Notwendigkeit, von gesetzwidrigem Verwaltungshandeln in ihren Rechten verletzten Personen dadurch eingetretene Schäden zu ersetzen. ${ }^{{ }_{21}}$ Dafür kennt das Grundgesetz nur die unzulängliche Haftung des Staates für Amtspflichtverletzungen seiner Beamten nach Art. 34 GG; ${ }^{* 22}$ ob weitergehende Rechtsfolgen bei Untätigbleiben des Gesetzgebers unmittelbar aus dem Verfassungsrecht folgen, ist fraglich. ${ }^{{ }^{23}}$ Derzeit behilft man sich daher mit vom Ende des 18.Jahrhunderts stammenden, angeblichen Gewohnheitsrechtssätzen zur Aufopferung. ${ }^{*}{ }^{24}$

\section{c) Zum Vorbehalt des Gesetzes}

Durch den Vorbehalt des Gesetzes sind bestimmte Entscheidungen der Gesetzgebung ,vorbehalten', damit der Verwaltung verboten, wenn sie nicht durch Gesetz vom Verbot freigestellt und zum Handeln ermächtigt wird. Der überkommene Anwendungsbereich des Vorbehalts sind seit dem 19. Jahrhundert Eingriffe in Freiheit und Eigentum des Bürgers; verstanden als Chiffre für die gesamte individuelle Rechtssphäre des Bürgers ist dies bis heute der zentrale Gegenstand des Vorbehalts. ${ }^{25}$ Dadurch ist für die gesamte Eingriffs-

18 M. Sachs. § 31: Verfahrensfehler im Verwaltungsverfahren. - W. Hoffmann-Riem, E. Schmidt-Aßmann, A. Voßkuhle (Hrsg.). Grundlagen des Verwaltungsrechts. Bd. II: Informationsordnung, Verwaltungsverfahren, Handlungsformen. 2. Aufl. München: Beck 2012, Rdn. 73 ff.; M. Sachs. Zur formellen Rechtswidrigkeit von Verwaltungsakten. - Verwaltungsarchiv (VerwArch) 2006, S. $577 f f$.

19 Vgl. §§ 45, 46 VwVfG und dazu M. Sachs. § 31: Verfahrensfehler im Verwaltungsverfahren. - W. Hoffmann-Riem, E. SchmidtAßmann, A. Voßkuhle (Hrsg.) (Fn. 17), Rdn. 46.

20 Nach dem Grundsatz ,volenti non fit iniuria', dazu nur OVG Niedersachsen, Urteil v. 12.7.2001, 10 L 3954/99. - Niedersächsische Verwaltungsblätter (NdsVBl) 2001, 316 (317); M. Sachs. ,Volenti non fit iniuria'. - VerwArch 1985, S. 412 ff.

$21 \quad$ Vgl. M. Sachs (Fn. 2), Art. 20 Rdn. 111 m.w.N.

22 Das am 26. Juni 1981 erlassene und zum 1. Januar 1982 in Kraft getretene ,Staatshaftungsgesetz’ wurde sehr bald wegen Mangels der Gesetzgebungskompetenz für nichtig erklärt, vgl. BVerfG, Urteil v. 19.10.1982, 2 BvF 1/81, Staatshaftungsgesetz. - BVerfGE 61, 149 ff.; die 1994 geschaffene Bundesgesetzgebungskompetenz nach Art. 74 Abs. 1 Nr. 25 GG ist bis heute nicht genutzt worden.

23 Dagegen etwa M. Sachs (Fn. 2), vor Art. 1 Rdn. 42 und Art. 20 Rdn. 111 jeweils m.w.N.

24 L. Osterloh. § 55: Kompensation der Folgen rechtmäßigen Hoheitshandelns. - W. Hoffmann-Riem, E. Schmidt-Aßmann, A. Voßkuhle (Hrsg.). Grundlagen des Verwaltungsrechts. Bd. III: Personal, Finanzen, Kontrolle, Sanktionen, Staatliche Einstandspflichten. 2. Aufl. München: Beck 2013, Rdn. 48 ff;; W. Kluth. - H. J. Wolff, O. Bachof, R. Stober, W. Kluth (Hrsg.). Verwaltungsrecht II. 7. Aufl. München: Beck 2010, § 72 Rdn. 75ff.; H. Maurer (Fn. 6), § 28 Rdn. 1 ff.

25 Darüber hinaus wird der Vorbehalt auf andere (vor allem für die Grundrechte), wesentliche' Regelungen erstreckt, vgl. dazu nur M. Sachs (Fn. 2), Art. 20 Rdn. $116 f f$. 
verwaltung, für jedes den Bürger belastende Verwaltungshandeln, eine gesetzliche Grundlage erforderlich. Ausreichend dafür ist jedes, auch nur materielle Gesetz; die durch Demokratie und Gewaltenteilung gebotene maßgebliche Rückführung auf das formelle Gesetz ist dadurch sichergestellt, dass untergesetzliche Rechtsnormen (Verordnungen, aber in der Sache auch Satzungen) nur aufgrund einer hinreichend bestimmten formell-gesetzlichen Ermächtigung ergehen dürfen.

\section{Kontrollen der Gesetzmäßigkeit der Verwaltung}

Rechtliche Garantien sind im Ernstfall nur so viel wert wie ihre Kontrolle. Dies gilt auch und gerade für die Gesetzmäßigkeit der Verwaltung.

\section{a) Objektive Kontrollmechanismen}

Eine besondere Institution, die - wie wohl der Justizkanzler in Estland nach Art. 139 ff. der Verfassung umfassend die Gesetzmäßigkeit der Verwaltung zu sichern hätte, gibt es in Deutschland nicht. Insbesondere ist der Bundesrechnungshof nach Art. 114 GG nur in Bezug auf die Ordnungsmäßigkeit der Haushalts- und Wirtschaftsführung (auch) zur Kontrolle der Rechtmäßigkeit berufen, nicht aber in Bezug auf das gesamte Verwaltungshandeln. ${ }^{*}{ }^{26}$ Nur partikulare Kontrollaufgaben haben verfassungsrechtlich vorgesehene Beauftragte ${ }^{* 27}$ wie der Wehrbeauftragte nach Art. $45 \mathrm{~b} \mathrm{GG}^{* 28}$, der Datenschutzbeauftragte, wie ihn etwa Art. 77a der Verfassung Nordrhein-Westfalens vorsieht, ${ }^{* 29}$ oder der Bürgerbeauftragte nach Art. 36 der Verfassung des Landes Mecklenburg-Vorpommern.

Im Übrigen gehört zur verfassungsrechtlichen Aufgabe der Mitglieder der Regierungen in Bund und Ländern, ihren jeweiligen Geschäftsbereich zu leiten, ${ }^{*}{ }^{30}$ insbesondere die Aufsicht in rechtlicher Hinsicht. ${ }^{*}{ }^{11}$ Für die Ausführung der Bundesgesetze durch die Länder ist die Aufsicht jedenfalls über deren Rechtmäßigkeit in Art. 84 Abs. 3, 4 und Art. 85 Abs. 4 GG ausdrücklich angesprochen. Die grundsätzlich lückenlose Rechtsaufsicht über staatliche Behörden wie über selbständige Verwaltungsträger, auch solche mit Selbstverwaltung, ist ein Gebot rechtsstaatlicher Organisation. ${ }^{*}{ }^{32}$

\section{b) Garantie des Rechtswegs gegen die öffentliche Gewalt}

Neben diesen objektiven Sicherungen und ungeachtet des Beitrags, den auch das Petitionsgrundrecht des Art. 17 GG leisten kann, ist von zentraler Bedeutung die in Art. 19 Abs. 4 GG garantierte Möglichkeit, gegen die öffentliche Gewalt, und das heißt hier jedenfalls im Kern: gegen die Verwaltung, ${ }^{*} 33$ den Rechtsweg zu beschreiten, also unabhängige Gerichte anzurufen. Dieses Recht steht aber - unbeschadet weitergehender Möglichkeiten, die das Gesetz eröffnen kann und ggf. kraft Unionsrechts eröffnen muss - nur denjenigen zu, die die Verletzung ihrer eigenen individuellen Rechte geltend machen können. Die Wahrung der Gesetzmäßigkeit der Verwaltung tritt nur reflexweise ein; das eigentliche Anliegen der Rechtsweggarantie ist der Schutz der subjektiven Rechte betroffener Personen. Soweit diese nicht zumal als Grundrechte von Verfassungs wegen bestehen, liegt ihre Reichweite allerdings in der Hand des Gesetzgebers, der die Verwaltung rechtlichen Bindungen unterwerfen kann, ohne entsprechende Individualrechte zu begründen. ${ }^{*} 34$

26 H. Siekmann. - M. Sachs (Hrsg.) (Fn. 2), Art. 114 Rdn. 13, 28.

27 Dazu allgemein P. J. Tettinger. § 111 Die Beauftragten. - J. Isensee, P. Kirchhof (Hrsg.). Handbuch des Staatsrechts der Bundesrepublik Deutschland. Bd. V: Rechtsquellen, Organisation, Finanzen. 3. Aufl. Heidelberg: Müller 2007.

28 Er ist nach Satz 1 der Bestimmung insbesondere zum ,Schutz der Grundrechte' (nach der gesetzlichen Regelung des Näheren in $\S 1$ Abs. 3 Satz 1 Wehrbeauftragtengesetz: der Grundrechte der Soldaten) bestellt.

29 Nach $\S 22$ Abs. 1 Datenschutzgesetz (DSG) NRW „überwacht [er] die Einhaltung der Vorschriften dieses Gesetzes sowie anderer Vorschriften über den Datenschutz bei den öffentlichen Stellen.”

30 S. nur Art. 65 Satz 2 GG und Art. 55 Abs. 2 Verfassung des Landes Nordrhein-Westfalen.

31 S. Schiedermair. § 48 Selbstkontrollen der Verwaltung. - W. Hoffmann-Riem, E. Schmidt-Aßmann, A. Voßkuhle (Hrsg.) (Fn. 17), Rdn. 23.

32 M. Sachs (Fn. 2), Art. 20 Rdn. 161 m.w.N.

33 Zur umstrittenen Reichweite der Rechtsweggarantie gegenüber Gesetzgebung und Rechtsprechung s. nur Ibid., Art. 19 Rdn. 120 ff. m.w.N.

34 Dazu und zur dafür maßgeblichen Schutznormlehre nur Ibid., Art. 19 Rdn. 129ff. m.w.N. 
Die verfassungsgerichtliche Rechtsprechung hat den Art. 19 Abs. 4 GG in viele Richtungen zu einer Garantie auch effektiven Rechtsschutzes ausgebaut. Einzelheiten sind hier nicht zu referieren. ${ }^{*} 35$ Erwähnt sei mit Blick auf die Bedeutung für das Verwaltungshandeln nur, dass die Kontrolle grundsätzlich in rechtlicher wie tatsächlicher Hinsicht umfassend sein muss, ${ }^{*}{ }^{36}$ dass es Vorwirkungen auf das Verwaltungsverfahren in Bezug auf Begründung und Dokumentation mit Blick auf die Durchführbarkeit der Gerichtskontrolle geben kann ${ }^{*} 37$ und dass die Verwaltung mit Behinderungen ihres Wirkens durch Verfahren des einstweiligen und des vorläufigen Rechtsschutzes rechnen muss. ${ }^{*} 8$

\section{Allgemeine inhaltliche Anforderungen an das Verwaltungshandeln}

Der Rechtsstaat erschöpft sich unter dem Grundgesetz nicht in den überkommenen formellen Garantien von Gesetzmäßigkeit und Gerichtsschutz, sondern enthält auch materielle, inhaltliche Elemente.

\section{a) Gerechtigkeit}

$\mathrm{Zu}$ den allgemeinen inhaltlichen Vorgaben gehört zum einen die Bindung an fundamentale Anforderungen der Gerechtigkeit. Sie kann neben anderen Aspekten in der Bindung der Rechtsanwendung an Gesetz und Recht gesehen werden, ${ }^{*} 39$ im Rahmen verfassungsgemäßer Gesetze wird sie allerdings im Wesentlichen als Leitgedanke der Gesetzesanwendung bedeutsam.

\section{b) Verhältnismäßigkeit}

Der heute praktisch überragend wichtige Grundsatz der Verhältnismäßigkeit ist in einigen neueren Landesverfassungen explizit angesprochen, ${ }^{*} 40$ für das Grundgesetz unabhängig von einer Stütze im Wortlaut durch die Verfassungsrechtsprechung als gegenüber der gesamten Staatsgewalt von Verfassungs wegen wirksames Element der Rechtsstaatlichkeit entwickelt worden. ${ }^{*}{ }^{11}$ Schon gegenüber den Gesetzen, ggf. auch im Wege verfassungskonformer Auslegung, wirksam, erlangt der Grundsatz unmittelbare Bedeutung für die Verwaltung nur, soweit diese gesetzesfrei handelt oder im Rahmen der Gesetzesanwendung über Spielräume verfügt. Dann muss die Verwaltung die Aspekte der Eignung, der Erforderlichkeit und der Proportionalität der von ihr zur Erreichung ihrer legitimen Ziele eingesetzten Mittel unabhängig von diesbezüglichen besonderen Gesetzen beachten.

\section{c) Vertrauensschutz}

Der Vertrauensschutz tritt im Grundgesetz zumal im Verbot der Bestrafung bei ex post facto gesetzlich bestimmter Strafbarkeit, Art. 103 Abs. 2 GG (nulla poena sine lege praevia), hervor, ${ }^{*}{ }^{4}$ auch den Strafklageverbrauch nach Art. 103 Abs. 3 GG (ne bis in idem) kann man dem Vertrauensschutz verpflichtet sehen. ${ }^{*} 4$

Weitergehend ist der Schutz individuellen Vertrauens gegenüber dem Staatshandeln als subjektive Kehrseite des objektiven Anliegens der Rechtssicherheit ganz allgemein ein Element des Rechtsstaatsprinzips. Seine genaue Reichweite gegenüber der Verwaltung festzulegen, ist insbesondere im Konflikt mit

35 Dazu nur Ibid., Art. 19 Rdn. 143ff. m.w.N.

36 Ibid., Art. 19 Rdn. 145 m.w.N.

37 Ibid., Art. 19 Rdn. 143a m.w.N.

Ibid., Art. 19 Rdn. $148 f f$. m.w.N.

Ibid., Art. 20 Rdn. 103.

40 Vgl. nach der Wiedervereinigung Art. 5 II 1, 23 II Verfassung des Landes Brandenburg; Art. 20 II 1 Verfassung des Landes Sachsen Anhalt; Art. 42 IV 1 Verfassung des Freistaats Thüringen.

41 S. schon BVerfG, Urteil v. 3.6.1954, 1 BvR 183/54, Zulassung politischer Parteien zur Landtagswahl. - BVerfGE 3, 383 (399); ständige Rechtsprechung seit BVerfG, Urteil v. 11.6.1958, 1 BvR 596/56, Apotheken. - BVerfGE 7, 377 (405, $407 f$.); vgl. etwa Beschluss v. 9.3.1994, 2 BvL 43/92, Cannabis. - BVerfGE 90, 145 (173).

42 S. etwa C. Degenhart. - M. Sachs (Hrsg.) (Fn. 2), Art. 103 Rdn. 54 m.w.N.

43 Dafür ausdrücklich etwa G. Nolte. - H. v. Mangoldt, F. Klein, C. Starck (Hrsg.). Kommentar zum Grundgesetz . Bd. 3. 6. Aufl. München: Vahlen 2010, Art. 103 Rdn. 179 m.w.N. 
anderen Anliegen der Verfassung, zumal dem der Gesetzmäßigkeit der Verwaltung, der Gesetzgebung überlassen. Diese hat insbesondere den Widerruf und die Rücknahme von begünstigenden Verwaltungsakten Einschränkungen mit Rücksicht auf den Vertrauensschutz unterworfen (vgl. §§ 48, 49 VwVfG); doch kann in diesem Bereich ergänzend ${ }^{*} 44$ wie auch in anderen Zusammenhängen* 45 der Schutz von Vertrauen auch verfassungs-unmittelbar durchgreifen.

\section{Vorgaben für Organisation und Verfahren der Verwaltung}

Aus dem Rechtsstaatsprinzip werden auch Vorgaben für Organisation (zu a) und Verfahren (zu b) der Verwaltung abgeleitet.

\section{a) Organisatorische Anforderungen}

Schon erwähnt wurde, dass die Gesetzmäßigkeit der Verwaltung durch Strukturen der Rechtsaufsicht gesichert werden muss (oben zu 2 a). Dies gilt nicht nur innerhalb der staatlichen Behördenhierarchie und gegenüber verselbständigten Trägern öffentlicher Verwaltung, sondern muss auch beim Einsatz privatrechtlicher Organisationsformen durchgreifen. ${ }^{*} 46$ Eine Flucht ins Privatrecht vor den besonderen Anforderungen an das Verhalten der öffentlichen Gewalt darf es auch jenseits der Grundrechtsbindung nicht geben. ${ }^{*} 47$

Ferner lässt sich der Funktionsvorbehalt des Art. 33 Abs. 4 GG zugunsten eines durch die hergebrachten Grundsätze des Berufsbeamtentums nach Art. 33 Abs. 5 GG geprägten öffentlichen Dienstes jedenfalls auch als rechtsstaatliche Sicherung der Gesetzmäßigkeit der Verwaltung lesen. Der besondere Status der Lebenszeitbeamten gibt diesen - ähnlich der persönlichen Unabhängigkeit der Richter - eine Basis, sich auch gegenüber Anmutungen von Vorgesetzten oder gleich der politischen Führung für eine gesetzestreue Aufgabenwahrnehmung zu entscheiden. ${ }^{*} 48$ Auch das Gebot der Bestenauslese nach Art. 33 Abs. 2 GG kann man als (freilich in der Praxis bis heute kaum effektives) Mittel gegen Parteienpatronage im öffentlichen Dienst ${ }^{*} 49$ hierher zählen.

Bedeutsam für die Verwaltung ist schließlich das in Art. 33 Abs. 5 GG verankerte Streikverbot der Beamten, ${ }^{*}{ }^{\circ}$ das allerdings ins Blickfeld des Europäischen Gerichtshofs für Menschenrechte geraten ist. ${ }^{*} 5^{1}$ Daher könnte zumindest seine uneingeschränkte Fortgeltung in Frage gestellt sein.

44 M. Sachs. - P. Stelkens, H. J. Bonk, M. Sachs (Hrsg.) (Fn. 13), § 48 Rdn. 182 ff.

45 Vgl. zur Möglichkeit einer auf Vertrauensschutz gegründeten Selbstbindung der Verwaltung M. Sachs. - P. Stelkens, H. J. Bonk, M. Sachs (Hrsg.) (Fn. 13), § 40 Rdn. 104; zur zugunsten der Betroffenen durchgreifenden Verwirkung behördlicher Rechte Ibid., $\S 53$ Rdn. $21 f f$.

46 S. Schiedermair. § 48 Selbstkontrollen der Verwaltung. - W. Hoffmann-Riem, E. Schmidt-Aßmann, A. Voßkuhle (Hrsg.) (Fn. 17), Rdn. 47; H. Dreier. Hierarchische Verwaltung im demokratischen Staat. Tübingen: Mohr Siebeck 1991, S. 252 ff.

47 BVerfG, Urteil v. 22.02.2011, 1 BvR 699/o6, Fraport. - BVerfGE 128, 226 (245); BGH, Urteil v. 17.06.2003, XI ZR 195/O2. - Entscheidungen des Bundesgerichtshofs in Zivilsachen (BGHZ) 155, 166 (175f.); M. Burgi. § 18 Rechtsregime. W. Hoffmann-Riem, E. Schmidt-Aßmann, A. Voßkuhle (Hrsg.) (Fn. 6), Rdn. 45ff.; W. Hoffmann-Riem. § 33 Rechtsformen, Handlungsformen, Bewirkungsformen. - Ibid., Rdn. 110 ff.; H. Maurer (Fn. 6), § 3 Rdn. 26.

48 BVerfG, Beschluss v. 3.7.1985, 2 BvL 16/82, Schulleiterfunktion. - BVerfGE 70, 251 (267); Bayerischer Verfassungsgerichtshof, Entscheidung v. 26.10.2004, Vf. 15-VII-01. - NVwZ-RR 2005, 830 (831); P. Badura. - T. Maunz, G. Dürig (Begründer). Grundgesetz. München: Beck (Losebl.-Ausg.), Art. 33 (2010) Rdn. 67.

49 M. Sachs. - K. Stern. Das Staatsrecht der Bundesrepublik Deutschland. Bd. IV/2: Die einzelnen Grundrechte. München: Beck 2011, S. 1844; W. Höfling. - W. Kahl, C. Waldhoff, C. Walter (Hrsg.) (Fn. 15), Art. 33 Abs. 1 bis 3 (2007) Rdn. $146 f f$.

50 U. Battis. - M. Sachs (Hrsg.) (Fn. 2), Art. 33 Rdn. 71; H. Lecheler. § 110 Der öffentliche Dienst. - J. Isensee, P. Kirchhof (Hrsg.) (Fn. 4), Rdn. 43.

51 Vgl. namentlich Europäischer Gerichtshof für Menschenrechte (EGMR), Urteil v. 21.4.2009, 68959/o1. - Neue Zeitschrift für Arbeitsrecht (NZA) 2010, 1423ff.; demgegenüber OVG Nordrhein-Westfalen, Urteil v. 7.3.2012, 3d A 317/11.O. - NVwZ 2012, 890 ff.; zur Problematik ferner etwa H.-W. Laubinger. - M. Breuer, A. Epiney, A. Haratsch, S. Schmahl, N. Weiß (Hrsg.). Der Staat im Recht. Festschrift für Eckart Klein zum 70. Geburtstag. Berlin: Duncker \& Humblot 2013, S. 1141 ff.; T. Hebeler. Noch einmal: Gibt es ein Streikrecht für Beamte? - Zeitschrift für Beamtenrecht (ZBR) 2012, S. 325 ff.; C. Traulsen. Das Beamtenstreikverbot zwischen Menschenrechtskonvention und Grundgesetz. - Juristenzeitung (JZ) 2013, S. 65ff. - DOI: http://dx.doi.org/10.1628/002268813X13560134286993 


\section{b) Anforderungen an das Verwaltungsverfahren}

Zu rechtsstaatlichen Anforderungen an das Verwaltungsverfahren wurde bereits auf die Vorwirkungen hingewiesen, die sich mit Rücksicht auf eine möglicherweise nachfolgende gerichtliche Überprüfung ergeben. Darüber hinaus sind rechtsstaatliche Desiderate wie Sicherungen der Neutralität der befassten Amtswalter, die Gewährung von Gehör vor Eingriffen der Verwaltung oder die Begründung von Verwaltungsakten zu nennen, die heute in den Verwaltungsverfahrensgesetzen verankert sind, ohne dass die Reichweite ihrer verfassungsrechtlichen Notwendigkeit im Einzelnen klar wäre. ${ }^{* 2}$ Aus dem Wesen rechtsstaatlicher Planung wird das auch verfahrensbezogene Gebot einer umfassenden Abwägung abgeleitet. ${ }^{*} 3$

Die Frage der Relativierung von Verfahrensfehlern wurde schon angesprochen. ${ }^{*} 54$ Dahingehende Regelungen stehen zwar nicht in Widerspruch zum Vorrang des Gesetzes, weil Verfahrensfehler überhaupt nur dann die sog. formelle Rechtswidrigkeit des inhaltlich nicht gesetzwidrigen Verwaltungsaktes begründen, wenn eine negative Rechtsanerkennungsnorm die Rechtswidrigkeit als Rechtsfolge der Verfahrensfehler besonders anordnet. ${ }^{*} 5$ Dies ist im Falle absoluter Verfahrensfehler stets der Fall, im Übrigen nach den überkommenen Rechtsprechungsgrundsätzen nur dann, wenn der Verfahrensfehler den Inhalt der Verwaltungsentscheidung beeinflusst haben kann. ${ }^{*}{ }^{6}$ Doch bleibt diese Folgenlosigkeit von Verfahrensfehlern für die Rechtmäßigkeit des Verwaltungsakts ebenso bedenklich wie die bei Rechtswidrigkeit verbleibenden Möglichkeiten der Heilung noch im Gerichtsverfahren und des Ausschlusses des Aufhebungsanspruchs nach $\S \S 45,46$ VwVfG, solange auch keine anderen wirksamen Sanktionen an die Rechtswidrigkeit geknüpft sind. ${ }^{*} 57$

\section{Grundrechtliche Determinanten öffentlicher Verwaltung}

Auch die Grundrechte sind für die öffentliche Verwaltung von großer Bedeutung; Art. 1 Abs. 3 spricht die Bindung der vollziehenden Gewalt (also auch der Regierung und des Militärs) an die Grundrechte als unmittelbar geltendes Recht ausdrücklich aus. Während den Grundrechten im ausgehenden 19. Jahrhundert unmittelbare Rechtsgeltung ${ }^{*}{ }^{8}$ gerade für die Verwaltung beigemessen wurde, ist dies inzwischen nur noch in Grenzen der Fall.

Wichtigster Adressat der Grundrechtsbindung ist heute die Gesetzgebung. Sie schafft dem Verwaltungshandeln Raum gegenüber dem Vorbehalt des Gesetzes und damit gegenüber den Grundrechten als Abwehrrechten, muss aber selbst den Grundrechten genügen. Soweit der Grundrechtsbindung entsprechende Gesetze das Verwaltungshandeln in der Sache wie in Organisation und Verfahren abschließend determinieren, bleibt die unmittelbare Grundrechtsbindung der Verwaltung weithin ohne selbständige Bedeutung. ${ }^{*} 59$ Immerhin können die Grundrechte in diesem Bereich die verfassungsorientierte, ggf. verfassungskonforme Auslegung ${ }^{*} 60$ der Gesetze gebieten, die dann primär von der Verwaltung zu leisten ist.

Unmittelbar bedeutsam für die Verwaltung bleiben die Grundrechte aber vor allem, wo das Gesetz dem Verwaltungshandeln Spielräume lässt. Namentlich hat die Verwaltung das ihr eingeräumte Ermessen unter Berücksichtigung grundrechtlicher Vorgaben auszufüllen, namentlich Rücksicht zu nehmen auf

52 Vgl. nur M. Sachs (Fn. 2), Art. 20 Rdn. 165. Zu von einzelnen Verwaltungsverfahren unabhängigen Informationsrechten s. unten zu Fn. 69.

53 Zuletzt BVerwG, Urteil v. 10.10.2012, 6 C 36/11. - BVerwGE 144, 284 Rdn. 41 m.w.N., auch zur materiellen Seite.

54 Oben zu 1 a.

55 Näher M. Sachs. - P. Stelkens, H. J. Bonk, M. Sachs (Hrsg.) (Fn. 13), § 45 Rdn. 6; M. Sachs. - VerwArch 2006, S. $578 f f$. m.w.N.; auch M. Sachs. - W. Hoffmann-Riem, E. Schmidt-Aßmann, A. Voßkuhle (Hrsg.) (Fn. 17), § 31 Rdn. $24 f f$.

56 Vgl. zu den allein die Rechtswidrigkeit begründenden wesentlichen Verfahrensfehlern M. Sachs. - P. Stelkens, H. J. Bonk, M. Sachs (Hrsg.) (Fn. 13), § 45 Rdn. 117 ff. m.w.N.

57 M. Sachs. - W. Hoffmann-Riem, E. Schmidt-Aßmann, A. Voßkuhle (Hrsg.) (Fn. 17), § 31 Rdn. 113ff.

58 Im Gegensatz zu auf die Gesetzgebung zielenden programmatischen Grundrechtsgehalten; dazu K. Stern (Fn. 13), S. 1182 ff.; M. Sachs. - K. Stern (Fn. 48), S. 481 ff. jeweils m.w.N.

59 Während in diesem Bereich die Beachtung der Gesetze eine Verletzung der Grundrechte ausschließt, bleibt eine Verletzung des Gleichheitssatzes möglich, wenn in Parallelfällen gesetzwidrig gehandelt wurde. Zu dieser unter der Chiffre von dem vielfach zu pauschal geleugneten Recht auf Gleichheit im Unrecht behandelten Problematik vgl. Ibid., S. 1591ff.

6o Zu diesen von verfassungsrechtlichen Einflüssen bestimmten Auslegungsformen vgl. nur M. Sachs (Fn. 2), Einf. Rdn. 52 ff. 
grundrechtlich geschützte Interessen Betroffener und die stets angesprochenen Gleichheitsgrundrechte zu beachten. Aus diesen kann sich eine Selbstbindung der Verwaltung an ihre bisherige Übung ergeben, die allerdings Umstellungen der Praxis für die Zukunft nicht ausschließt. Ähnliches gilt im Falle von Beurteilungsspielräumen im Rahmen gesetzlicher Tatbestände. ${ }^{*} 61$

Die Grundrechte behalten ihre Bedeutung auch, wenn dieVerwaltung in privatrechtlichen Organisationsformen tätig wird; eine Flucht vor der Grundrechtsbindung ist auf diesem Wege nicht möglich.

\section{Demokratische Determinanten öffentlicher Verwaltung}

Das Demokratieprinzip entfaltet seine Bedeutung für die Verwaltung in inhaltlicher Hinsicht maßgeblich durch die schon als Rechtsstaatselement behandelte Bindung an das Gesetz, das seinerseits unmittelbar oder zumindest mittelbar auf den Willen des Volkes zurückzuführen ist. Soweit das Gesetz Spielräume lässt, wird durch den hierarchischen Aufbau der Verwaltung sichergestellt, dass die ihrerseits dem jeweiligen Parlament verantwortliche Regierung die Entscheidungsinhalte determinieren kann. ${ }^{* 62}$

Neben dieser inhaltlichen demokratischen Legitimation wird dem Grundgesetz die Notwendigkeit personeller demokratischer Legitimation entnommen, die im Rahmen der staatlichen Verwaltungen dadurch sichergestellt wird, dass die in der Verwaltung tätigen Personen durch die Regierungsmitglieder für ihr jeweiliges Ressort bestellt werden. Die Minister werden ihrerseits unmittelbar von der Volksvertretung gewählt oder durch den so gewählten Regierungschef bestimmt.

Problematisch sind demgegenüber sog. ministerialfreie Räume, also Bereiche, die dem Weisungsrecht der Regierung und damit deren Verantwortung gegenüber der Volksvertretung entzogen sind. ${ }^{* 6}$ Soweit sie nicht verfassungsrechtlich besonders legitimiert sind, wie etwa öffentlich-rechtliche Rundfunkanstalten durch die Rundfunkfreiheit des Art. 5 Abs. 1 Satz 2 GG $^{*}{ }^{64}$ oder auf Landesebene verfassungsrechtlich verankerte unabhängige Datenschutzbeauftragte, ${ }^{*}{ }_{5}$ begegnen sie Bedenken. Nach Unionsrecht vorgesehene unabhängige Stellen dürften allerdings kraft dessen Anwendungsvorrangs trotz mangelnder demokratischer Legitimation als zulässig angesehen werden. ${ }^{* 6}$

Probleme mit der demokratischen Legitimation bestehen auch bei der Selbstverwaltung: Diese ist allerdings auf der kommunalen Ebene mit der insoweit in Art. 28 Abs. 2 GG vorgeschriebenen Volksvertretung als Sonderform orts-demokratischer Legitimation verfassungsrechtlich gut ableitbar. In den zahlreichen Fällen nur gesetzlich begründeter funktionaler Selbstverwaltung durch Beteiligte eines Lebensbereichs - von den Kammern freier Berufe über Sozialversicherungsträger oder Universitäten ${ }^{*} 67$ bis zu

61 Zum ganzen nur M. Sachs. - P. Stelkens, H. J. Bonk, M. Sachs (Hrsg.) (Fn. 13), § 40 Rdn. $165 f f$.

62 H. Dreier (Fn. 3), Art. 20 (Demokratie) Rdn. 124; H. Dreier (Fn. 45), S. 129 ff., 141 ff.; F. Kirchhof. - T. Maunz, G. Dürig (Begründer) (Fn. 47), Art. 83 (2009) Rdn. $58 f$.

63 BVerfG, Urteil v. 27.4.1959, 2 BvF 2/58, Bremer Personalvertretung. - BVerfGE 9, 268 (282); M. Sachs (Fn. 2), Art. 20 Rdn. 41; K. Stern. Das Staatsrecht der Bundesrepublik Deutschland. Bd. II: Staatsorgane, Staatsfunktionen, Finanz- und Haushaltsverfassung, Notstandsverfassung. München: Beck 1980, S. 790 f; H. Dreier (Fn. 3), Art. 20 (Demokratie) Rdn. $126 f$; ; B. Grzeszick. - T. Maunz, G. Dürig (Begründer) (Fn. 47), Art. 20 II (2010) Rdn. 196 ff.; E. Klein. Die verfassungsrechtliche Problematik des ministerialfreien Raumes. Berlin: Duncker \& Humblot 1974.

64 M. Sachs (Fn. 2), Art. 20 Rdn. 41; F. Kirchhof. - T. Maunz, G. Dürig (Begründer) (Fn. 47), Art. 83 (2009) Rdn. $28 f$.

65 Wie nach Art. 77a Verfassung des Landes Nordrhein-Westfalen. Allerdings unterliegen Regelungen der Landesverfassungen über das Homogenitätsgebot des Art. 28 Abs. 1 Satz 1 GG den Grundsätzen des insbesondere demokratischen Rechtsstaates im Sinne des Grundgesetzes, die aber bei eng begrenzte punktuelle Abweichungen dieser Art nicht verletzt sein dürften; vgl. zur ,eingeschränkten Regelungsdichte' des Homogenitätsprinzips M. Nierhaus. - M. Sachs (Hrsg.) (Fn. 2), Art. 28 Rdn. 10-12. Das Bundesverfassungsgericht scheint unabhängige Datenschutzbeauftragte geradezu für verfassungsrechtlich notwendig zu halten, vgl. BVerfG, Urteil v. 15.12.1983, 1 BvR 209/83 u.a., Volkszählung. - BVerfGE 65, 1 (46); BverfG, Urteil v. 2.3.2010, 1 BvR 256/o8 u.a., Vorratsdatenspeicherung. - BVerfGE 125, 260 (327).

66 Dies gilt jedenfalls, solange nicht geradezu das durch Art. 23 Abs. 1 Satz 3, Art. 79 Abs. 3 GG geschützte Demokratieprinzip als solches berührt würde, vgl. allgemein BVerfG, Urteil v. 30.6.2009, 2 BvE 2/o8 u.a., Lissabon-Vertrag. - BVerfGE 123, 267 ( 356 ff.); zur Unberührbarkeit der geschützten Grundsätze M. Sachs (Fn. 2), Art. 79 Rdn. 32 ff.

67 Für diese bestehen zum Teil landesverfassungsrechtliche Sonderregelungen zugunsten ihrer Selbstverwaltung, so etwa in Art. 16 Verfassung des Landes Nordrhein-Westfalen. 
Wasserverbänden - ist es hingegen nicht immer unproblematisch, das notwendige Maß an demokratischer Legitimation festzustellen. ${ }^{*} 68$

Eine Notwendigkeit plebiszitärer Einwirkungsmöglichkeiten auf die Verwaltung, wie sie gesetzlich auf kommunaler Ebene vorgesehen sind, sind jedenfalls nach dem Grundgesetz und der Landesverfassung Nordrhein-Westfalen nicht gefordert. Auch die zuletzt in das Verwaltungsverfahrensgesetz (§ 25 Abs. 3) aufgenommene erweiterte Beteiligung der (betroffenen) Öffentlichkeit ist nicht vom Demokratieprinzip geboten. Dagegen wird die Öffentlichkeit des Staats- und insbesondere Verwaltungshandelns als Element von Demokratie angesehen, was wegen privater und öffentlicher Interessen notwendiger Geheimhaltung nicht entgegensteht; ${ }^{*} 99$ inzwischen ist dieses Anliegen in Informationsfreiheitsgesetzen auf Bundes- und Landesebene aufgegriffen worden. ${ }^{*} 70$

Auch unter demokratischem Blickwinkel anzusprechen sind schließlich Formen formeller Privatisierung öffentlicher Verwaltung; diese dürfen nicht dazu führen, dass das Verwaltungshandeln dem maßgeblichen Einfluss der dem Parlament verantwortlichen Regierung bzw. der legitimierten kommunalen Leitungsorgane entzogen wird. ${ }^{*} 1$

\section{Schlussbemerkung}

Insgesamt lässt sich danach festhalten, dass die öffentliche Verwaltung in Deutschland heute in hohem Maße durch das Verfassungsrecht bestimmt ist. Abgesehen von den spezifisch bundesstaatlichen Determinanten sind es zumal Elemente des Rechtsstaatsprinzips und die Grundrechte, nur begrenzt auch das Demokratieprinzip, die für den Aufbau der öffentlichen Verwaltung und ihr Tätigwerden von ganz fundamentaler Bedeutung sind, zumeist allerdings vermittelt durch die verfassungsgemäßen Gesetze. In diesem

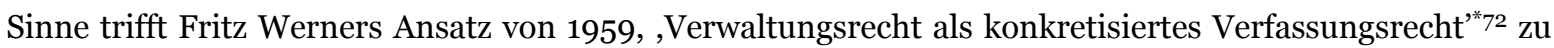
verstehen, zu.

Die gern damit konfrontierte Aussage Otto Mayers von 1924, ,Verfassungsrecht vergeht, Verwaltungsrecht besteht', 73 mag nach 1919 gestimmt haben; schon nach 1933 (dazu wurde gestern berichtet), dann nach 1949, zuletzt nach 1989 mit der Ablösung des sowjetisch installierten kommunistischen Systems für die Deutsche Demokratische Republik (DDR) und die aus ihr hervorgegangenen Länder sowie aufgrund der Einflüsse des Unionsrecht hat sich aber auch das Verwaltungsrecht mit den angesprochenen verfassungsrechtlichen Umwälzungen ${ }^{*} 74$ maßgeblich fortentwickelt.

Trotz allen Wandels gibt es aber in Verfassungsrecht und Verwaltungsrecht doch eine allerdings nicht ununterbrochene Kontinuität; denn seit dem 19. Jahrhundert fortdauerndes rechtsstaatliches Urgestein, zumal die Gesetzmäßigkeit der Verwaltung und der auch gegen sie eröffnete gerichtliche Rechtsschutz, gehört auch heute zu den zentralen verfassungsrechtlichen Determinanten der öffentlichen Verwaltung in Deutschland.

68 Vgl. zu den nicht stets einheitlichen Maßstäben der bundesverfassungsgerichtlichen Judikatur nur M. Sachs (Fn. 2), Art. 20 Rdn. 44a; ablehnend zu abweichenden Legitimationsmodellen zuletzt etwa B. Grzeszick. Die Voraussetzungen demokratischer Legitimation der Verwaltung und deren Konzeptualisierung in der Verfassungsrechtsdogmatik. - M. Anderheiden u.a. (Hrsg.). Verfassungsvoraussetzungen. Gedächtnisschrift für Winfried Brugger. Tübingen: Mohr Siebeck 2013, S. 601 ff.

69 Vgl. allgemein nur M. Sachs (Fn. 2), Art. 20 Rdn. 18 m.w.N.

70 Zum verfassungsrechtlichen Hintergrund nur F. Schoch. Informationsfreiheitsgesetz. München: Beck 2009, Einl. Rdn. $6 f f$.

71 M. Sachs (Fn. 2), Art. 20 Rdn. 39 m.w.N.

72 DVBl. 1959, S. $527 f f$.

73 O. Mayer. Deutsches Verwaltungsrecht. Bd. I. 3. Aufl. Leipzig: Duncker \& Humblot 1924, Vorwort S. VI.

74 Die Veränderungen aufgrund des Unionsrechts, wenngleich nicht selbst verfassungsrechtlicher Natur, sind durch verfassungsrechtliche Vorgaben des Grundgesetzes zumindest ermöglicht worden. 